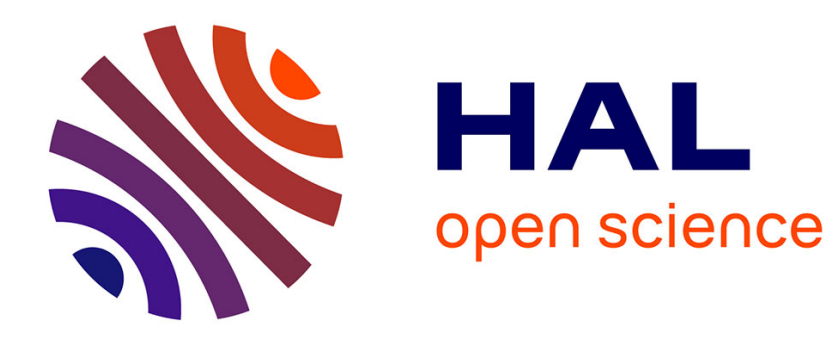

\title{
Tunable Optical Vortex Arrays from a Single Nematic Topological Defect
}

\author{
Etienne Brasselet
}

\section{To cite this version:}

Etienne Brasselet. Tunable Optical Vortex Arrays from a Single Nematic Topological Defect. Physical Review Letters, 2012, 108 (8), pp.087801. 10.1103/PhysRevLett.108.087801 . hal-00676572

\section{HAL Id: hal-00676572 \\ https://hal.science/hal-00676572}

Submitted on 11 Apr 2018

HAL is a multi-disciplinary open access archive for the deposit and dissemination of scientific research documents, whether they are published or not. The documents may come from teaching and research institutions in France or abroad, or from public or private research centers.
L'archive ouverte pluridisciplinaire HAL, est destinée au dépôt et à la diffusion de documents scientifiques de niveau recherche, publiés ou non, émanant des établissements d'enseignement et de recherche français ou étrangers, des laboratoires publics ou privés. 


\title{
Tunable Optical Vortex Arrays from a Single Nematic Topological Defect
}

\author{
Etienne Brasselet* \\ Univ. Bordeaux, LOMA, UMR 5798, F-33400 Talence, France \\ CNRS, LOMA, UMR 5798, F-33400 Talence, France \\ (Received 20 September 2011; published 23 February 2012)
}

\begin{abstract}
We report on the generation of tunable structured light fields endowed with various sets of phase singularities from a single topological defect in a nematic liquid crystal mesophase. The experimental demonstration relies on the use of electric field-induced nonsingular topological defects called "umbilics."
\end{abstract}

In wave physics, phase singularities correspond to location in space where the phase of a wave is undefined and represent a generic manifestation of interferences of at least three plane waves. In optics, this either occurs randomly, as in speckle fields [1], or in a controlled manner with the aim at designing, for instance, arrays of phase singularities [2]. The control of optical phase singularities actually forms the basis for applications such as optical information processing, contactless manipulation of matter from atomic to microscopic scale, optical threedimensional (3D) structuring of materials, super-resolution microscopy or astronomical imaging [3]. Besides the routine use of computer generated holograms to produce optical phase singularities with arbitrary topological charge [4], smart designs based on ordered soft-matter systems emerged as an attractive solution thanks to the wealth of existing self-organized structures, among which topological defects are prime choice ones. Indeed material topological defects can drive "regular" light beams (i.e., fields with smooth spatial profiles) into "singular" ones like optical vortex beams, whose name refers to twisted flow of light around a phase singularity [5].

Some demonstrations relying on the diffraction from dislocations have been made in liquid crystals [6] and colloidal crystals [7]. More recently, examples exploiting the geometric Pancharatnam-Berry phase [8] have been reported in the case of topological defects in nematic liquid crystal droplets [9] and films [10]. However experiments are restricted so far to the generation of a single optical vortex with fixed topological charge. In addition, several methods have been implemented to write, at the centimeter scale, various 2D patterns for the optical axis distribution in uniaxial mesogenic materials. Such patterns can bear topological defects with arbitrary topological charge [11], or even arrays of them [12]. Nevertheless, the latter strategies turn out to be restricted to the design of singular macroscopic optical elements since the control of the topology at the microscale remains an open issue (e.g. see Fig. 3 in [13]). Still, recent experiments have demonstrated the possibility to induce anisotropic microstructures endowed with topological defects using focused light beams in achiral [14] or chiral [15] nematics. However, when explored, their use is restricted to the generation of charge two phase singularities [16].

Here we show the possibility to generate arrays of optical vortices from a fixed single nematic liquid crystal topological defect, in a controllable manner and at the microscale $(10-100 \mu \mathrm{m})$. In particular, we demonstrate the control of (i) the number of vortices in a given array, (ii) the radial and azimuthal spatial location of the vortices, and (iii) the operation mode at the desired wavelength. This is made possible by combining electrooptical properties of nematics to the singular optical features of 3D orientational structures called "umbilical defects", in short "umbilics" [17]. These are nonsingular topological defects that appear in nematic films with perpendicular alignment and negative dielectric anisotropy, which were early reported in $[18,19]$.

Umbilics appear above the electric field Fréedericksz transition threshold voltage $U=U_{F}$ and possess a topological strength $s= \pm 1$. They are associated to a director field (a unit vector $\mathbf{n}$ defined as the local averaged molecular orientation) that changes not only along the $z$ axis (defined as the normal to the film) but also along the radial $(r)$ and azimuthal $(\phi)$ polar coordinates in the $(x, y)$ plane [17]. The 3D structure of the director is depicted in Figs. 1(a) and 1(b) in the case of a $s=+1$ umbilic of the splay type [17], which is however without lack of generality in the present work. The director can be represented in the Cartesian coordinates system associated with the orthonormal basis $(\mathbf{x}, \mathbf{y}, \mathbf{z})$ in the form

$$
\mathbf{n}=(\sin \vartheta \cos \varphi, \sin \vartheta \sin \varphi, \cos \vartheta),
$$

where $\varphi$ is the azimuthal angle of the director in the $(x, y)$ plane whereas $\vartheta$ refers to its tilt angle with the $z$ axis. Following [17], umbilics are described by

$$
\begin{gathered}
\varphi=s \phi+\phi_{0}, \\
\vartheta(r, z)=\vartheta_{\infty} a(r) \sin (\pi z / L),
\end{gathered}
$$

where $\phi_{0}$ is a constant, $L$ is the film thickness, $0 \leq a \leq 1$ is the reduced tilt amplitude and $\vartheta_{\infty}$ is the asymptotic value of $\vartheta$ at large $r$, whose expression is given by 

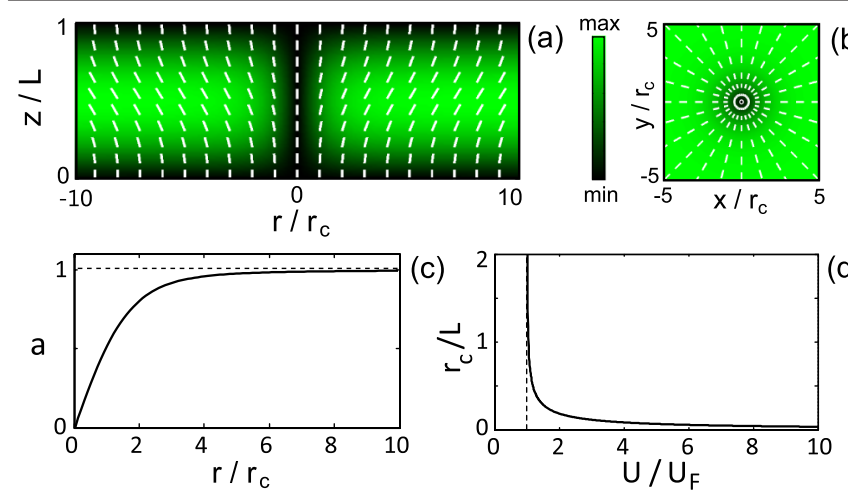

(d)

FIG. 1 (color online). Typical meridional [panel (a)] and equatorial $[z=L / 2$, panel (b)] cross sections of the director field of a $s=+1$ umbilic of the splay type; $r_{c}$ is the core radius of the defect and $L$ is the nematic film thickness. The color scale refers to the amplitude of the transverse part of $\mathbf{n}$, i.e., $\sin \vartheta$, see Eq. (1), whereas the white segments represent the local orientation of the director. (c) Calculated reduced amplitude $a$ of the tilt angle $\vartheta$ nearby the defect located at $r=0$. (d) Calculated reduced core radius $r_{c} / L$ vs reduced voltage $\tilde{U}$.

$$
\vartheta_{\infty}=\left[2\left(\tilde{U}^{2}-1\right) /\left(K_{1} / K_{3}-\tilde{U}^{2} \varepsilon_{a}^{\Omega} / \varepsilon_{\|}^{\Omega}\right)\right]^{1 / 2},
$$

where $\tilde{U}=U / U_{F}$ is the reduced voltage, $\varepsilon_{a}^{\Omega}=\varepsilon_{\|}^{\Omega}-\varepsilon_{\perp}^{\Omega}$ is the dielectric anisotropy at frequency $\Omega$ with $(\|, \perp)$ referring to directions along and perpendicular to $\mathbf{n}$, and $K_{1}$ and $K_{3}$ are the splay and bend Frank elastic constants. Finally, the reduced amplitude satisfies

$$
\frac{d^{2} a}{d \rho^{2}}+\frac{1}{\rho} \frac{d a}{d \rho}+\left(1-\frac{1}{\rho^{2}}\right) a-a^{3}=0
$$

with the boundary conditions $a(0)=0$ and $a(\infty)=1 ; \rho=$ $r / r_{c}$ is the reduced radial coordinate, $r_{c}=(L / \pi) \times$ $\left(\mathcal{K} / K_{3}\right)^{1 / 2}\left(\tilde{U}^{2}-1\right)^{-1 / 2}$ being the core radius of the defect, where $\mathcal{K}$ is the effective elastic constant associated to a given umbilic [17]. Equation (5) is solved numerically, see Fig. 1(c), and the reduced core radius $r_{c} / L$ vs the reduced voltage is shown in Fig. 1(d). The 3D orientational structure of umbilics has already been determined experimentally by fluorescence confocal polarizing microscopy in [20] and next we show how these defects can be used to generate optical vortex arrays in a controllable manner.

In experiments, we used the nematic liquid crystal MLC-2079 (from Licristal) with $\varepsilon_{a}^{\Omega}=-6.1, \varepsilon_{\|}^{\Omega}=4.1$ and $\varepsilon_{\perp}^{\Omega}=10.2$ at $\Omega=1 \mathrm{kHz}, \quad K_{1}=15.9 \mathrm{pN}, \quad K_{3}=$ $18.3 \mathrm{pN}$, and refractive indices along and perpendicular to the nematic director are $n_{\|}=1.64$ and $n_{\perp}=1.49$ at $589 \mathrm{~nm}$ wavelength. The sample is a $30 \mu \mathrm{m}$-thick film sandwiched between two glass substrates provided with transparent electrodes in the visible. We found $U_{\mathrm{F}} \simeq 1.90 \mathrm{~V}_{\mathrm{rms}}(\Omega=2 \mathrm{kHz}$ in experiments $)$ that agrees within few percents to the predicted value $U_{\mathrm{F}}=$ $\pi\left[K_{3} /\left(\varepsilon_{0}\left|\varepsilon_{a}^{(\Omega)}\right|\right)\right]^{1 / 2}, \varepsilon_{0}$ being the vacuum permittivity, which gives $U_{\mathrm{F}}=1.83 \mathrm{~V}_{\mathrm{rms}}$ using the data sheet information from the manufacturer. In practice, an isolated umbilical defect is obtained in the following manner. First a voltage $U \gg U_{\mathrm{F}}$ is applied, which leads to the generation of a dense assembly of umbilics with topological strength $s= \pm 1$. Then, a annihilation dynamical process between defects with opposite strength takes place [21]. At the end of the process it turns out that few umbilics remains at fixed location over the $\sim 1 \mathrm{~cm}^{2}$ area of the film, from which we select one. Its observation between parallel linear polarizers reveals the generation of a growing number of optical vortex quadrupoles as the applied voltage is increased. This is illustrated in Fig. 2(a) that corresponds to an incident light with $\lambda=532 \mathrm{~nm}$ wavelength and linear polarization state along the $x$ axis. In this figure, $A_{m}$ refer to the array made of $m$ quadrupoles, $A_{m}=\left\{Q_{1}, Q_{2}, \ldots, Q_{m}\right\}$, where $Q_{n}$ is the $n$th optical vortex quadrupole starting numbering from the central part of the defect here located in the center of each panel. In addition, in Fig. 2, the voltage value is set to observe a maximal and almost uniform transmission far away from the defect, a situation to which we further refer to as the "bright field" case. In practice, this is found to occur at $\tilde{U}_{m}^{\exp }=$ $(1.12,1.25,1.37,1.51)$ for $m=(1,2,3,4)$, respectively.

Observations are confronted with simulations in Fig. 2(b), which is obtained in the following manner. First we define the electric field of the incident linearly polarized light as $\mathbf{E}_{\text {in }}=E_{0} e^{-i\left(\omega t-k_{0} z\right)} \mathbf{x}$, where $\omega$ is the angular frequency of light and $k_{0}=2 \pi / \lambda$ is the wave vector in free space. Also, we introduce the total phase delay $\Delta$ acquired between the extraordinary and ordinary waves when light has passed throughout the film,

$$
\Delta(r)=\frac{2 \pi}{\lambda} \int_{0}^{L}\left[n_{e}(r, z)-n_{\perp}\right] d z,
$$

where $n_{e}$ is the effective local refractive index of the extraordinary wave,

$$
n_{e}(r, z)=\frac{n_{\|} n_{\perp}}{\left[n_{\|}^{2} \cos ^{2} \vartheta(r, z)+n_{\perp}^{2} \sin ^{2} \vartheta(r, z)\right]^{1 / 2}} .
$$

Then, apart an unimportant phase factor, the output electric field expression is calculated, which gives
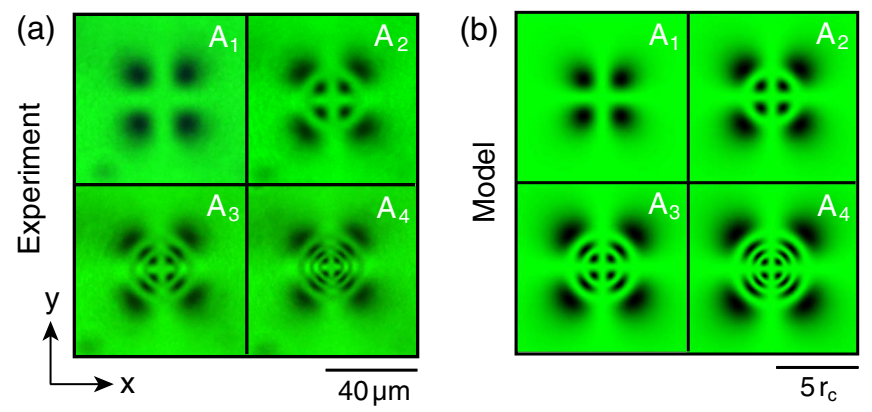

FIG. 2 (color online). (a) Experimental intensity patterns of the optical transmission between parallel linear polarizers (here the passing direction is set along the $x$ axis) when $\tilde{U}$ is chosen to fulfill the bright field condition for the optical vortex arrays $A_{m}$, $m=(1,2,3,4)$ (see text for details). (b) Corresponding intensity patterns calculated from Eq. (9). 
$A_{1}$

(a)

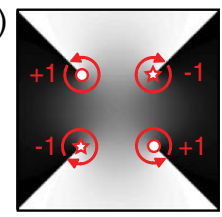

(b)

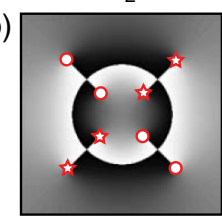

$\mathrm{A}_{3}$

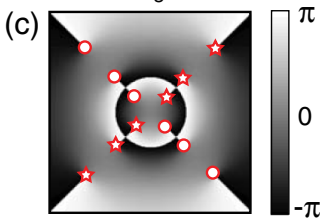

FIG. 3 (color online). Calculated phase profile of the output light field under monochromatic illumination and parallel polarizers imaging for bright field arrays $A_{m}$ with $m=1$ (a), 2 (b), and 3 (c). The location of individual single charge optical vortex is indicated by circles (topological charge +1 ) and stars (topological charge -1 ). The calculated region of interest corresponds to a $6 r_{c} \times 6 r_{c}$ area.

$$
\begin{aligned}
& \mathbf{E}_{\text {out }}(r, \varphi) \propto E_{0}(\{\cos [\Delta(r) / 2]+i \cos (2 \varphi) \sin [\Delta(r) / 2]\} \mathbf{x} \\
&+i \sin (2 \varphi) \sin [\Delta(r) / 2] \mathbf{y}) .
\end{aligned}
$$

Finally, the intensity and phase profiles of the copolarized output light field component with respect to the input polarization state are derived from Eq. (8),

$I_{\text {out }}^{\pi_{\|}}(r, \varphi)=I_{0}\left(\cos ^{2}[\Delta(r) / 2]+\cos ^{2}(2 \varphi) \sin ^{2}[\Delta(r) / 2]\right)$,

with $I_{0}$ the maximum output intensity value and

$$
\Phi_{\text {out }}^{\pi_{\|}}(r, \varphi)=\arctan \{\tan [\Delta(r) / 2] \cos (2 \varphi)\},
$$

where the superscript $\pi_{\|}$refers to copolarized imaging under linearly polarized illumination.

On the one hand, Eq. (9) allows to identify the condition for the observation of the above-mentioned bright field arrays $A_{m}$ as $\Delta_{\infty}=\Delta(r \rightarrow \infty)=2 m \pi, m$ integer. The corresponding voltage values $\tilde{U}_{m}$ are therefore numerically found by solving $\Delta_{\infty}=2 m \pi$ from Eqs. (3), (4), (6), and (7), imposing $a=1$. A satisfactory qualitative agreement with experimental data is obtained; see Fig. 2(b). Unfortunately,

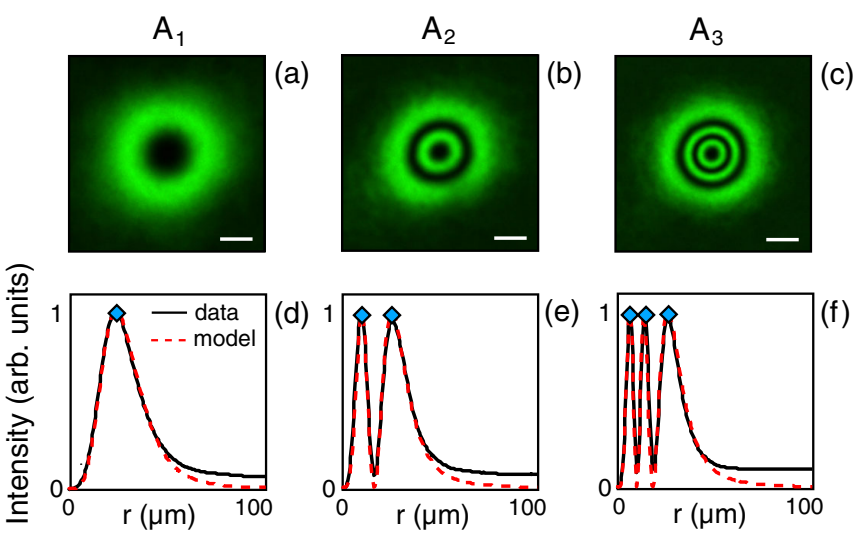

FIG. 4 (color online). Upper row: transmission between crossed circular polarizers at $\lambda=532 \mathrm{~nm}$ associated to the bright field arrays $A_{1}, A_{2}$, and $A_{3}$. Scale bar is $20 \mu \mathrm{m}$. Bottom row: confrontation between experimental data (solid line) and the model (dashed line) for the radial intensity profile under crossed circular polarizers. Diamonds markers refer to the condition $\Delta\left(r_{n}\right)=$ $(2 n+1) \pi$ where $r_{n}$ corresponds to the radius of the circle on which lies the four single charge optical vortices of $Q_{n}$. the quantitative comparison between experimental and calculated voltage values $\tilde{U}_{m}$ turns out to be a pointless attempt. To understand why, let us analyze the simplest case $m=1$. At first sight, the predicted value $\tilde{U}_{1}^{\text {model }}=1.19$ seems reasonably close to the experimental one, $\tilde{U}_{1}^{\exp }=$ 1.12. However, the corresponding director tilt angle is quite large, namely $\vartheta_{\infty}^{\text {model }}=0.53$, thereby emphasizing that a Fourier expansion of the director field along the $z$ axis truncated to the lowest mode [see the $\sin (\pi z / L)$ term in Eq. (3)] is a yet acceptable shortcoming. In addition, we note that the expected fourfold symmetry intensity pattern is slightly broken at large voltage, see for instance the pattern $A_{4}$ in Fig. 2(a) that shows a anisotropic lensing effect.

On the other hand, Eq. (10) reveals the claimed quadrupolar substructure of arrays $A_{m}$. This is shown in Fig. 3 where panels (a),(b) and (c) refer to the phase profile associated to $A_{1}, A_{2}$ and $A_{3}$, respectively. As an example, in the case $m=1$, there are four phase singularities with alternating topological charge +1 (circle symbols) and -1 (star symbols) that form the quadrupole $Q_{1}$; see Fig. 3(a). Similarly, optical vortex quadrupoles $Q_{2}$ and $Q_{3}$ can be identified in Fig. 3(b) and 3(c).

Experimentally, the phase structure is retrieved from the determination of $\Delta(r)$, see Eq. (10). This is achieved by measuring the intensity pattern obtained between crossed circular polarizers. Indeed, assuming an incident circularly polarized light $\mathbf{E}_{\text {in }}=E_{0} e^{-i\left(\omega t-k_{0} z\right)} \mathbf{c}_{ \pm}$, where $\mathbf{c}_{ \pm}=$ $(\mathbf{x} \pm i \mathbf{y}) / \sqrt{2}$ are the circular polarization basis unit vectors, and applying the same procedure as in the linear case above we get

$$
\mathbf{E}_{\text {out }}(r, \varphi) \propto E_{0}\left\{\cos [\Delta(r) / 2] \mathbf{c}_{ \pm}+i e^{ \pm 2 i \varphi} \sin [\Delta(r) / 2] \mathbf{c}_{\mp}\right\},
$$

from which we obtain the intensity of the contra-polarized output light field component,

$$
I_{\text {out }}^{\sigma_{\perp}}(r)=I_{0} \sin ^{2}[\Delta(r) / 2],
$$

where the superscript $\sigma_{\perp}$ refers to contra-polarized imaging under circularly polarized illumination. The corresponding experimental intensity patterns associated to the bright field arrays $A_{1}, A_{2}$ and $A_{3}$ are shown in Figs. 4(a)-4(c), respectively. The satisfactory confrontation between our observations and the model is illustrated in Figs. 4(d)-4(f) where
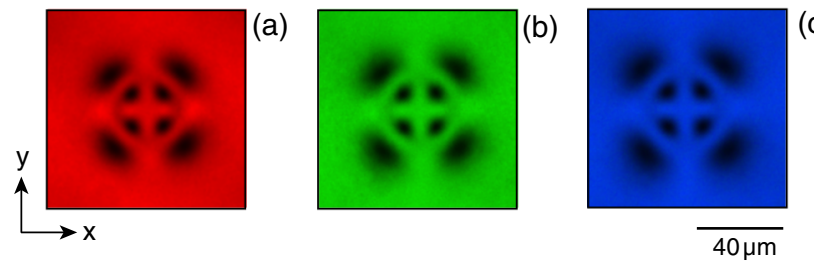

(c)

FIG. 5 (color online). Wavelength tunable bright field arrays illustrated in the case $m=2$ using red (R), green $(\mathrm{G})$, and blue (B) set of illumination wavelengths $\lambda_{\mathrm{R}}=632.8 \mathrm{~nm}$ (a), $\lambda_{\mathrm{G}}=$ $532 \mathrm{~nm}(\mathrm{~b})$, and $\lambda_{\mathrm{B}}=488 \mathrm{~nm}$ (c). 

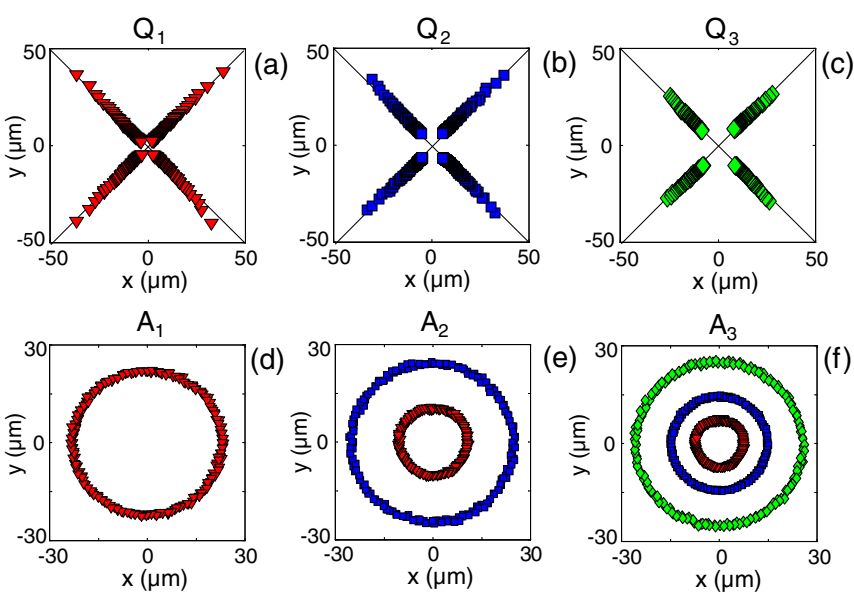

FIG. 6 (color online). Upper row: electrically-controlled radial position in the $(x, y)$ plane of quadrupoles $Q_{1}(\boldsymbol{\nabla}), Q_{2}(\boldsymbol{\square})$, and $Q_{3}(\diamond)$ for a $x$-polarized incident illumination at $\lambda=532 \mathrm{~nm}, \tilde{U}$ being varied from 1 to 1.6. Bottom row: polarization-controlled azimuthal position of the $m$ quadrupoles embedded in the bright field array $A_{m}$ when the angle $\alpha$ between the direction of the pair of parallel linear polarizers and the $x$ axis is varied from 0 to $\pi$. Quadrupoles labeling is the same as above.

the experimental normalized radial intensity profile (averaged over the azimuth) is superimposed with the theoretical profile given by Eq. (12).

The arrays $A_{m}$ can be electrically adjusted to any desired operating wavelength. This is illustrated in Fig. 5 in the particular case of the bright field array $A_{2}$ for red (R), green (G), and blue (B) illumination wavelengths $\lambda_{\mathrm{R}}=$ $632.8 \mathrm{~nm}$ [panel (a)], $\lambda_{\mathrm{G}}=532 \mathrm{~nm}$ [panel (b)] and $\lambda_{\mathrm{B}}=$ $488 \mathrm{~nm}$ [panel (c)], respectively. The corresponding reduced voltages are found to be $\tilde{U}_{2}^{\mathrm{R}}=1.33, \tilde{U}_{2}^{\mathrm{G}}=1.25$ and $\tilde{U}_{2}^{\mathrm{B}}=1.22$. Accordingly, the above-mentioned anisotropic lensing effect is all the more pronounced than the wavelength is large [see Fig. 5(a), along the $x$ axis] due to the $1 / \lambda$ dependence of the optical phase delay $\Delta$. Indeed, the larger is $\lambda$, the larger is the required voltage, hence the director reorientation amplitude, in order to fulfill the condition $\Delta_{\infty}=2 m \pi$, whatever is $m$.

Also, the radial and azimuthal location of the optical vortex quadrupoles embedded in the array $A_{m}$ can be controlled in the $(x, y)$ plane while the location of the umbilic is kept fixed. On the one hand, the electrically tunable radial position of the four single charge optical vortices that form the quadrupole $Q_{n}$ is shown in Fig. 6 for $n=1$ [panel (a)], $n=2$ [panel (b)] and $n=3$ [panel (c)]. As expected from Eqs. (9) and (10), the corresponding trajectories are straight lines at $\pm 45^{\circ}$ from the $x$ axis for a $x$-polarized incident light. On the other hand, the azimuthal control is achieved by varying the direction of the incident linearly polarized light whatever the array $A_{m}$. This is demonstrated in Fig. 6 for $m=1$ [panel (d)], $m=2$ [panel (e)] and $m=3$ [panel (f)] where the circular trajectories of each individual optical vortex is shown for the bright field arrays $A_{1}, A_{2}$ and $A_{3}$ when the azimuth $\alpha$ of the incident linear polarization state given by $\cos \alpha \mathbf{x}+$ $\sin \alpha \mathbf{y}$ is varied from 0 to $\pi$. In fact, such a behavior is expected from the rotational invariance around the $z$ axis of the optical retardance associated to the umbilic.

To conclude, the generation of optical vortex arrays at predetermined wavelength, with controlled number of individual optical vortex, combined with adjustable 2D positioning, has been demonstrated both experimentally and theoretically using umbilical defects in a nematic liquid crystal mesophase. In view of all existing kinds of topological defects in liquid crystals [22] these findings appear useful for the production of tailored complex light fields structures that can find applications, for instance, in optical imaging, trapping and micromanipulation.

*e.brasselet@loma.u-bordeaux1.fr

[1] K. O'Holleran, M. R. Dennis, F. Flossmann, and M. J. Padgett, Phys. Rev. Lett. 100, 053902 (2008).

[2] J. Becker, P. Rose, M. Boguslawski, and C. Denz, Opt. Express 19, 9848 (2011).

[3] D. L. Andrews, Structured Light and Its Applications: An Introduction to Phase-Structured Beams and Nanoscale Optical Forces (Academic Press-Elsevier, Burlington, 2008).

[4] J.E. Curtis, B. A. Koss, and D. G. Grier, Opt. Commun. 207, 169 (2002).

[5] J. Arlt, J. Mod. Opt. 50, 1573 (2003).

[6] D. Voloschenko and O. D. Lavrentovich, Opt. Lett. 25, 317 (2000).

[7] M. P. MacDonald, P. Prentice, and K. Dholakia, New J. Phys. 8, 257 (2006).

[8] G. Biener, A. Niv, V. Kleiner, and E. Hasman, Opt. Lett. 27, 1875 (2002).

[9] E. Brasselet, N. Murazawa, H. Misawa, and S. Juodkazis, Phys. Rev. Lett. 103, 103903 (2009).

[10] E. Brasselet and C. Loussert, Opt. Lett. 36, 719 (2011).

[11] S. C. McEldowney, D. M. Shemo, R. A. Chipman, and P. K. Smith, Opt. Lett. 33, 134 (2008).

[12] S. Nersisyan, N. Tabiryan, D. M. Steeves, and B. R. Kimball, Opt. Express 17, 11926 (2009).

[13] D. Mawet, E. Serabyn, K. Liewer, C. Hanot, S. McEldowney, D. Shemo, and N. O'Brien, Opt. Express 17, 1902 (2009).

[14] E. Brasselet, Opt. Lett. 34, 3229 (2009).

[15] I. I. Smalyukh, Y. Lansac, N. A. Clark, and R. P. Trivedi, Nature Mater. 9, 139 (2009).

[16] E. Brasselet, Phys. Rev. A 82, 063836 (2010).

[17] A. Rapini, J. Math. Phys. (N.Y.) 34, 629 (1973).

[18] R. B. Meyer, Mol. Cryst. Liq. Cryst. 16, 355 (1972).

[19] A. Saupe, Mol. Cryst. Liq. Cryst. 21, 211 (1973).

[20] M. Gu, I. I. Smalyukh, and O. D. Lavrentovich, Appl. Phys. Lett. 88, 061110 (2006).

[21] I. Dierking, O. Marshall, J. Wright, and N. Bulleid, Phys. Rev. E 71, 061709 (2005).

[22] M. Kleman, Rep. Prog. Phys. 52, 555 (1989). 